\title{
An atomic level study on the out-of-plane thermal conductivity of polycrystalline argon nanofilm
}

\author{
JU ShengHong \& LIANG XinGang* \\ Department of Engineering Mechanics, Key Laboratory for Thermal Science and Power Engineering of Ministry of Education, Tsinghua Univer- \\ sity, Beijing 100084, China
}

Received January 5, 2011; accepted August 31, 2011

\begin{abstract}
At present, there have been few direct molecular dynamics simulations on the thermal conductivity of polycrystalline nanofilms. In this paper, we generate polycrystalline argon nanofilms with random grain shape using the three-dimensional Voronoi tessellation method. We calculate the out-of-plane thermal conductivity of a polycrystalline argon nanofilm at different temperatures and film thicknesses by the Muller-Plathe method. The results indicate that the polycrystalline thermal conductivity is lower than that of the bulk single crystal and the single-crystal nanofilm of argon. This can be attributed to the phonon mean-free-path limit imposed by the average grain size as well as the grain boundary thermal resistance due to the existence many grain boundaries in polycrystalline materials. Also, the out-of-plane thermal conductivity of the polycrystalline argon nanofilm is insensitive to temperature and film thickness, and is mainly dominated by the grain size, which is quite different from the case of single-crystal nanofilms.
\end{abstract}

thermal conductivity, polycrystalline, argon nanofilm, molecular dynamics simulation

Citation: Ju S H, Liang X G. An atomic level study on the out-of-plane thermal conductivity of polycrystalline argon nanofilm. Chin Sci Bull, 2012, 57: 294-298, doi: $10.1007 / \mathrm{s} 11434-011-4787-2$

Molecular dynamics (MD) simulation has become a widely used method to investigate the thermal conductivity of single-crystal nanofilms during the last decade because of its low cost and high efficiency [1-5]. As an example, Lukes et al. [1] studied the feasibility of using the MD computational technique to predict the normal thermal conductivity of solid argon thin films, and their result shows that the thermal conductivity increases with the atomic layer number. They also found a modest over-prediction of thermal conductivity due to the over-stiffness of the Lennard-Jones potential when applied to solids. Feng et al. [2] investigated the thermal conductivity of argon films with thicknesses of 2-10 nm using nonequilibrium MD (NEMD). They found that the thin-film conductivity is observably lower than the bulk thermal conductivity and monotonously depends on the film thickness. Liu et al. [3] also observed an increase of the thermal conductivity of argon thin films with film

*Corresponding author (email: liangxg@tsinghua.edu.cn) thickness using NEMD. The increase rates are different for different film thickness ranges, and the size effect becomes negligible when the film thickness is beyond $100 \mathrm{~nm}$. They further studied the effect of vacancies on the argon film thermal conductivity and found that the vacancies can greatly reduce the film's conductivity by disrupting the regularity of the argon lattice.

Currently, polycrystalline materials attract many researchers' attention, because polycrystalline materials with nanoscale grain sizes are expected to have different properties compared with single crystals, such as mechanical properties, thermal properties, etc. [6,7]. Although MD has been widely used to predict the thermal conductivity of singlecrystal materials such as solid-argon nanofilm [1-3], few simulations on three-dimensional polycrystalline nanofilm have been reported. Zhong and Wang [8] constructed a simple polycrystalline argon model by putting several rectangular nano-grains together and calculated its thermal conductivity. Ju and Liang [9] simulated the thermal conduc- 
tivity of bulk polycrystalline solid argon with three-dimensional random grain shape at different temperatures using the Voronoi tessellation method. Their results demonstrate that the thermal property of polycrystalline materials can be investigated directly by MD simulation. In this paper, we report the simulation of the thermal conductivity of a polycrystalline argon nanofilm at different temperatures and film thicknesses by direct MD simulation using the threedimensional Voronoi tessellation method.

\section{Simulation method}

Solid argon is selected as the simulation material for its simple potential function, and because its thermal conductivity is also widely simulated or measured by researchers $[1-5,10]$. As argon is an inert element and a dielectric material, we only consider the phonon transport that dominates the thermal conductivity, without considering the electrons or their interactions with phonons. The argon atoms interact with each other through the classical Lennard-Jones (12-6) potential [11],

$$
\phi(r)=4 \varepsilon\left[\left(\frac{\sigma}{r}\right)^{12}-\left(\frac{\sigma}{r}\right)^{6}\right],
$$

where $r$ is the distance between a pair of atoms to be considered, $\varepsilon=1.67 \times 10^{-21} \mathrm{~J}$ is the well depth, and $\sigma=3.4 \times 10^{-10} \mathrm{~m}$ is the equilibrium separation. At the beginning of each simulation, a random kinetic energy drawn from the Boltzmann distribution for the desired system temperature is assigned to each atom in the computational region. The Verlet algorithm [11-13] is used to integrate the motion equations numerically. The integral time step $\Delta t$ is $1 \mathrm{fs}$. In the simulation, the system is first equilibrated in an NPT (fixed atom number $N$, pressure $P$ and temperature $T$ ) ensemble to achieve the controlled temperature and pressure. After equilibration, an $N V E$ (fixed atom number $N$, volume $V$ and energy $E$ ) ensemble is used. One simulation usually takes 2500000 steps, which is $2.5 \mathrm{~ns}$ in total. The first 500000 steps are used to make the system achieve a steady state, while the rest are used for statistical analysis. The Muller-Plathe (M-P) method [14-16], which is a NEMD method, is used to calculate the thermal conductivity.

We first use three-dimensional Voronoi tessellation to generate a polycrystalline argon configuration with random grain shape [17]. This method is extensively used in materials science, especially for modeling random microstructures like aggregates of grains in polycrystals [18-20]. For a set of points in the simulation domain, the tessellation is defined by assigning a cell space to each point. The atoms in the cell are closer to the point than to any other [18-20]. A Voronoi tessellation is a cell structure constructed from a Poisson point process by introducing planar cell walls perpendicular to the lines connecting the neighboring points.
For instance, to generate a polycrystalline argon structure, a set of points is first selected in the simulation box, and then equivalent grain cells are generated according to the definition of the Voronoi tessellation, as shown in Figure 1(a). After that, each cell is filled with argon atoms in a face-centered cubic lattice with a randomly selected crystal orientation, as shown in Figure 1(b). The atoms too close to each other at the grain boundaries are removed from the system before the simulation to reduce stress. More details of the polycrystalline structure generation process are described in [9]. The polycrystalline argon film structures are generated with periodic boundary conditions in three spatial directions according to the need of the M-P method to simulate the thermal conductivity.

In this paper, we use the M-P method [14-16] to obtain the thermal conductivity of polycrystalline argon nanofilms. This algorithm is also called the reverse NEMD approach. The conventional NEMD approach imposes a temperature gradient on the system and measures the response as a resulting heat flux. In M-P, the heat flux is imposed, and the temperature gradient is the system's response. The idea of M-P is to divide the simulation system into $N$ layers along the heat flux direction first, as shown in Figure 2. Then the hottest atoms in the first layer and the coldest atoms in the middle layer are chosen to exchange their velocities for every several steps, which induces a temperature gradient in the system. The thermal conductivity is calculated by [14]

$$
\lambda=d \frac{j_{z}}{\Delta T},
$$

where $d$ is the thickness of the nanofilm, $\Delta T$ is the temperature difference, and the heat flux $j_{z}$ is given by the sum of exchanged energy per unit time and area [14],

$$
j_{z}=\frac{1}{2 t A} \sum_{\text {exchange }} \frac{m}{2}\left(v_{\text {hot }}^{2}-v_{\text {cold }}^{2}\right),
$$

where $t$ is the simulation time, $A$ is the cross-sectional area of the simulation system perpendicular to the heat flux direction $z, m$ is the atom mass, $v_{\text {hot }}$ and $v_{\text {cold }}$ denote the velocities of the hottest and coldest atoms to be exchanged at each step, and the factor 2 arises from the periodicity. The simulation time is very important to the result. Usually, the

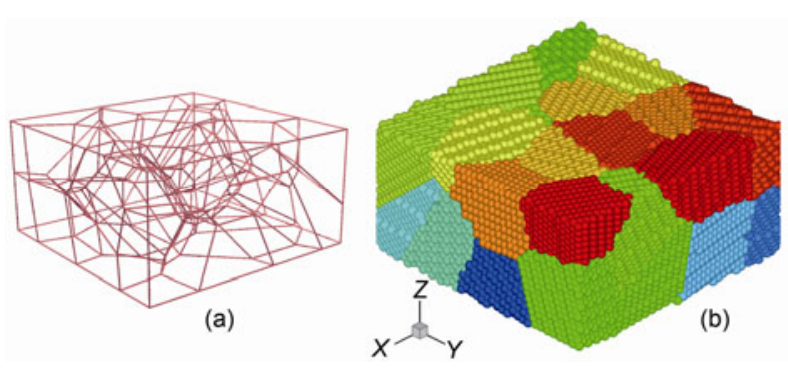

Figure 1 Sketch of (a) the Voronoi cells (grain cells) and (b) the polycrystalline argon nanofilm structure. 


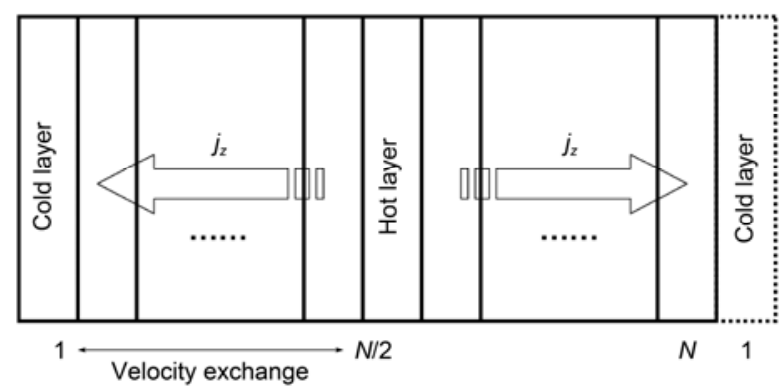

Figure 2 Sketch of the thermal conductivity calculated by the Muller-Plathe method.

temperature distribution obtained in the simulation time should be linear between the hot and cold film layers; otherwise, the simulation time should be increased. When the velocity exchange frequency is certain, the heat flux generated by the exchange increases almost linearly with the simulation time. So, the longer the time, the smaller the uncertainties of the heat flux. In this study, two nanoseconds is long enough to obtain a linear temperature distribution and a relatively steady heat flux. Figure 3 shows a typical temperature distribution by M-P in the argon nanofilm simulation. From the figure we find that the temperature distribution in the hot and cold layers is a bit nonlinear, because the velocities of the atoms in this region are exchanged manually to generate the temperature gradient. The linear temperature distribution between the hot and cold layers is used to calculate the film thermal conductivity.

\section{Results and discussion}

$\mathrm{Ju}$ and Liang [9] studied the system size effect in polycrystalline MD simulation and found that the periodic boundary effect can be ignored when the system size is two or three times as large as the grain size. Therefore, we set the cross-sectional area of the polycrystalline nanofilm as $2 D \times$ $2 D$ with periodic boundary conditions, where $D$ denotes the

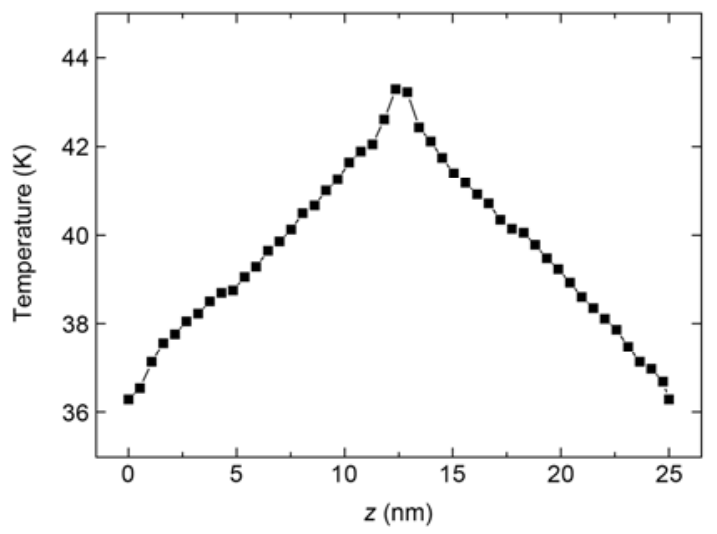

Figure 3 Typical temperature distribution obtained by the Muller-Plathe method. average grain size. The steadiness of the polycrystalline structure is very important during the simulation. Figure 4 illustrates the atom position in each grain of one polycrystal nanofilm configuration at different times during the whole simulation at $40 \mathrm{~K}$. From the figure we can see that the sharp edges of all the grains remain steady as designed, except that some atoms at the grain boundaries move a little bit from their initial positions. We also find that the atoms at grain boundaries move much more at higher temperature but less at low temperature. Comparing the atoms' positions at 0.5 and $2.5 \mathrm{~ns}$, few atoms change their positions further. The constructed polycrystal structure is seen to remain steady, which ensures that the thermal conductivity obtained is really the polycrystalline nanofilm thermal conductivity with controlled average grain size.

We first simulate the out-of-plane thermal conductivity of the polycrystalline nanofilm with thickness of $12.5 \mathrm{~nm}$ at temperatures from 20 to $70 \mathrm{~K}$. The average grain size is controlled at $5 \mathrm{~nm}$. A single-crystal film with the same thickness is also simulated at the same time for comparison. The results are shown in Figure 5. From the figure we see that the thermal conductivities of both the single-crystal and polycrystalline films are lower than that of single-crystal bulk argon, but the polycrystalline nanofilm thermal conductivity is much lower than that of the single-crystal film with the same thickness. The phonon mean-free-path (MFP) limit imposed by the average grain size as well as the grain boundary thermal resistance arising from the large amount
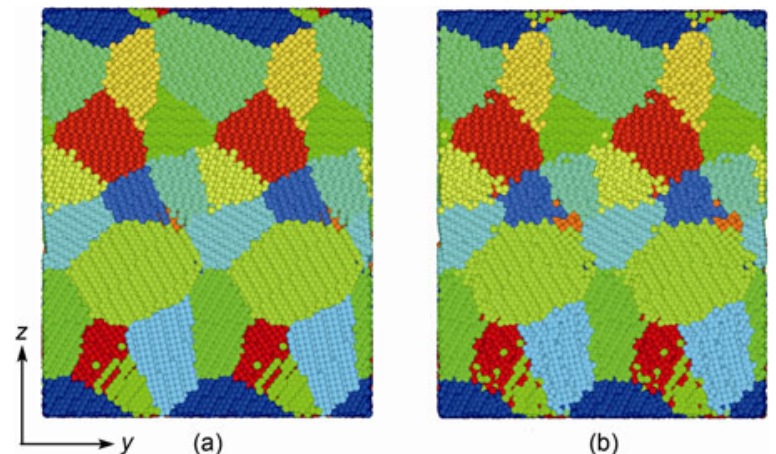

(b)

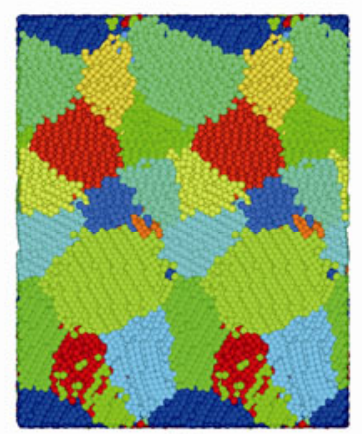

(c)

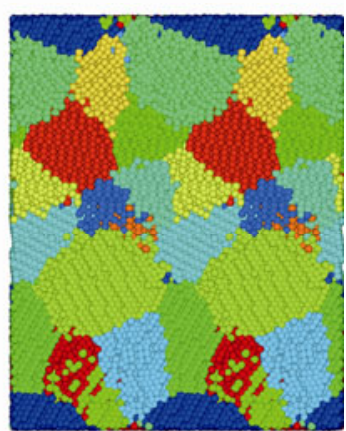

(d)

Figure 4 Atom positions of the polycrystalline argon nanofilm during one simulation, (a) at the initial moment, $t=0$; (b) at $t=0.5 \mathrm{~ns}$; (c) at $t=1.5 \mathrm{ns;}$ (d) at the end of the simulation $t=2.5 \mathrm{~ns}$. Direction $z$ is the film thickness direction. 


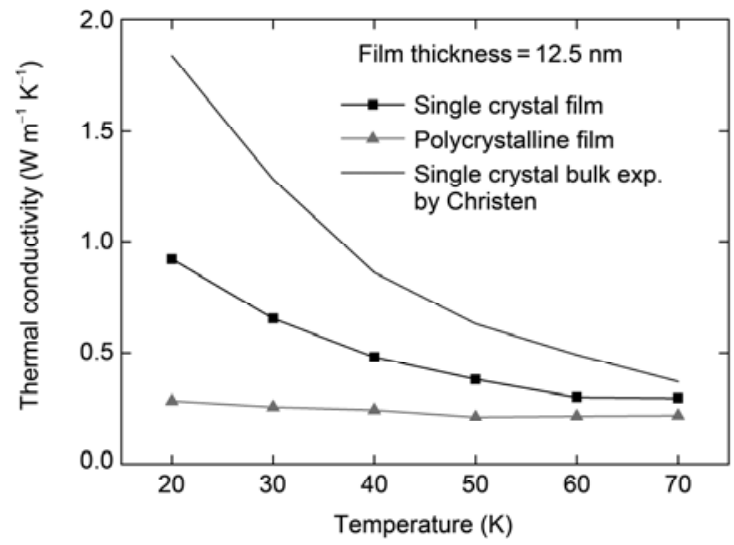

Figure 5 Thermal conductivity of the polycrystalline nanofilm at different temperatures.

of grain boundaries make the thermal conductivity of the polycrystalline nanofilm much smaller and insensitive to the temperature, as shown in Figure 5. However, the temperature dependence of the single-crystal thermal conductivity is clear because of the temperature dependence of the MFP.

We also use the M-P method to study the polycrystalline nanofilm thermal conductivity at film thickness from 12.5 to $32.5 \mathrm{~nm}$. The average temperature is controlled at $40 \mathrm{~K}$, and the average grain size is $5 \mathrm{~nm}$. The thermal conductivity of the single-crystal film is also simulated at the same time, and results are shown in Figure 6. The results are similar to the case of varying temperature. The thermal conductivities of both the single-crystal and polycrystalline nanofilms are lower than that of the bulk single crystal. The thermal conductivity of the polycrystalline film increases slightly as the thickness increases from 12.5 to $32.5 \mathrm{~nm}$, while that of the corresponding single-crystal film increases obviously.

Solid argon is a dielectric material, and phonons are the dominant energy carriers in heat transfer. So the MFP of phonons is a very important parameter. To understand the simulation results better, we estimate the phonon MFP of crystal argon. According to the thermal conductivity rela-

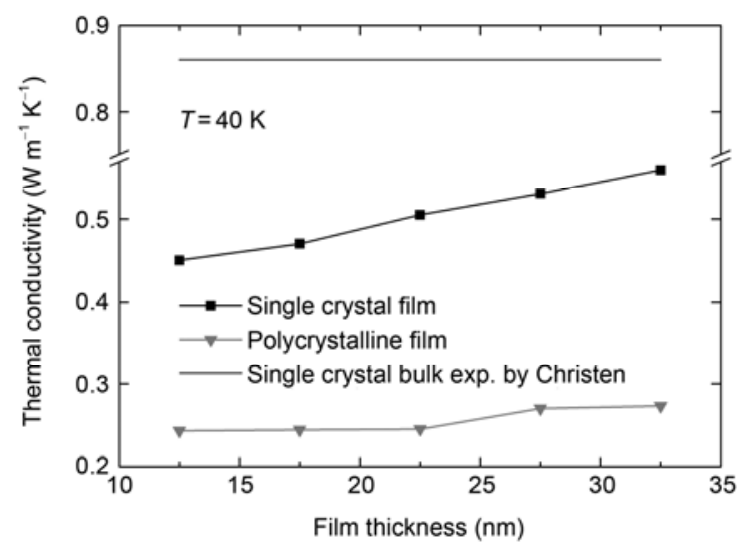

Figure 6 Thermal conductivity of the polycrystalline nanofilm at different film thicknesses. tion derived from kinetic theory $[21,22]$, the phonon MFP $l$ of bulk argon can be calculated as

$$
l=\frac{3 \lambda}{c v},
$$

where $\lambda$ is the thermal conductivity of bulk single-crystal argon, $c$ is the constant-volume specific heat capacity, and $v$ is the average sound velocity usually given by

$$
v=\frac{1}{3}\left(v_{L}+2 v_{T}\right),
$$

where $v_{L}$ is the longitudinal sound velocity and $v_{T}$ is the transverse sound velocity. The values of $\lambda, c, v_{L}, v_{T}$ used in the estimation are all from experiments [10,23,24]. The calculated phonon MFP of bulk argon is shown in Figure 7. As the temperature increases from 20 to $70 \mathrm{~K}$, the MFP of argon phonons drops from 9 to $1 \mathrm{~nm}$, which is comparable with the average grain size of $5 \mathrm{~nm}$.

Temperature and film thickness are two important parameters dominating the thermal conductivity of singlecrystal nanofilms. When temperature increases, the MFP in nanofilms decreases, and, as a result, the thermal conductivity decreases. When the single-crystal film thickness is comparable with the phonon MFP of the bulk crystal, the actual phonon MFP is dominated by the film thickness. The thinner the nanofilm is, the lower the thermal conductivity of single-crystal film is. When the grain size is comparable with the phonon MFP of the bulk material, the actual phonon MFP is dominated by the grain size, and the temperature and film thickness become much less important.

\section{Conclusions}

We used the three-dimensional Voronoi tessellation method to construct a polycrystalline argon nanofilm structure with random grain shape. We simulated the out-of-plane thermal conductivity of a polycrystalline nanofilm at different temper-

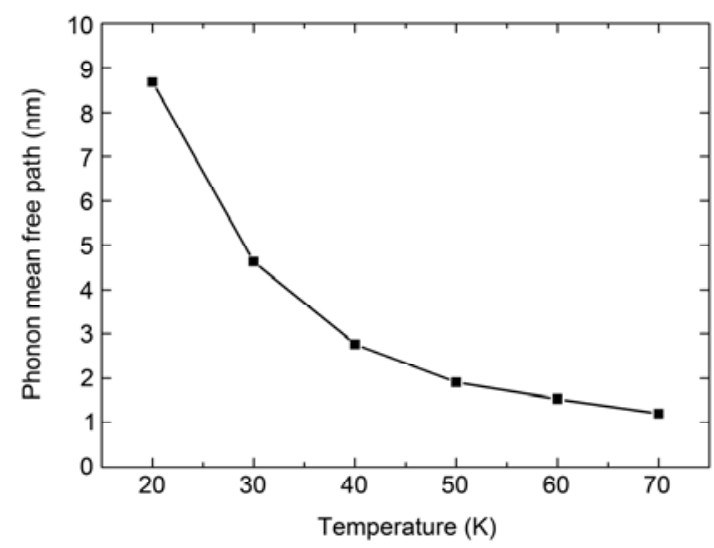

Figure 7 Phonon mean free path of bulk argon at different temperatures. 
atures and film thicknesses using the Muller-Plathe method. The out-of-plane thermal conductivity of polycrystalline argon nanofilm with more than one grain in the thickness direction is much lower than that of the corresponding single-crystal film with the same thickness. This can be attributed to the phonon MFP limit imposed by the average grain size as well as the grain boundary thermal resistance due to the large amount of grain boundaries in polycrystalline materials. The results also indicate that the thermal conductivity of a polycrystalline argon nanofilm changes little and can be considered constant for a wide range of temperature and film thickness. When the grain size in a polycrystalline nanofilm is comparable to or lower than the phonon MFP of bulk argon, the MFP is restricted by the average grain size, and the temperature and film thickness have little effect.

This work was supported by the National Natural Science Foundation of China (50776053 and 50730006).

1 Lukes J R, Li D Y, Liang X G, et al. Molecular dynamics study of solid thin-film thermal conductivity. J Heat Transf, 2000, 122: 536-543

2 Feng X L, Li Z X, Liang X G, et al. Molecular dynamics study on thermal conductivity of nanoscale thin films. Chin Sci Bull, 2001, 46: 604-608

3 Liu Q X, Jiang P X, Xiang H. Molecular dynamics study of the thermal conductivity of nanoscale argon films. Mol Simulat, 2006, 32: 645-649

4 Liang X G. Some effects of interface on fluid flow and heat transfer on micro- and nanoscale. Chin Sci Bull, 2007, 52: 2457-2472

5 Ju S H, Liang X G, Wang S C. Investigation of interfacial thermal resistance of bi-layer nanofilms by nonequilibrium molecular dynamics. J Phys D Appl Phys, 2010, 43: 085407

6 Soyez G, Eastman J A, Thompson L J, et al. Grain-size-dependent thermal conductivity of nanocrystalline yttria-stabilized zirconia films grown by metal-organic chemical vapor deposition. Appl Phys Lett, 2000, 77: 1155-1157
7 Meyers M A, Mishra A, Benson D J. Mechanical properties of nanocrystalline materials. Prog Mater Sci, 2006, 51: 427-556

8 Zhong Z, Wang X. Thermal transport in nanocrystalline materials. J Appl Phys, 2006, 100: 044310

$9 \mathrm{Ju} \mathrm{S} \mathrm{H}$, Liang X G. Investigation of argon nanocrystalline thermal conductivity by molecular dynamics simulation. J Appl Phys, 2010, 108: 104307

10 Christen D K, Pollack G L. Thermal conductivity of solid argon. Phys Rev B, 1975, 12: 3380-3391

11 Rapaport D C. The Art of Molecular Dynamics Simulation. New York: Cambridge University Press, 2004

12 Verlet L. Computer "experiment" on classical fluids. I. Thermodynamical properties of Lennard-Jones molecules. Phys Rev, 1967, 159: 98-103

13 Verlet L. Computer "experiment" on classical fluids. II. Equilibrium correlation functions. Phys Rev, 1967, 165: 201-214

14 Müller-Plathe F, Reith D. Cause and effect reversed in non-equilibrium molecular dynamics: An easy route to transport coefficients. Comput Theor Polym S, 1999, 9: 203-209

15 Müller-Plathe F. A simple nonequilibrium molecular dynamics method for calculating the thermal conductivity. J Chem Phys, 1997 , 106: 6082-6085

16 Müller-Plathe F. Reversing the perturbation in non-equilibrium molecular dynamics: An easy way to calculate the shear viscosity of fluids. Phys Rev E, 1999, 59: 4894-4899

17 Voronoi G. Nouvelles applications des paramètres continus à la théorie des formes quadratiques. J Reine Angew Math, 1907, 133: 97-178

18 Schiøtz J, Tolla F D D, Jacobsen K W. Softening of nanocrystalline metals at very small grain sizes. Nature, 1998, 391: 561-563

19 Rycroft C H, Grest G S, Landry J W, et al. Analysis of granular flow in a pebble-bed nuclear reactor. Phys Rev E, 2006, 74: 021306

20 Fritzen F, Böhlke T, Schnack E. Periodic three-dimensional meshgeneration for Voronoi tessellations with application to cubic crystal aggregates. Comput Mech, 2009, 43: 701-713

21 Ashcroft N W, Mermin N D. Solid State Physics. Philadephia: Sauders College, 1976

22 Ziman J M. Principles of the Theory of Solids. 2nd ed. London, New York, Melbourne: Cambridge University Press, 1979

23 Haenssler F, Gamper K, Serin B. Constant-volume specific heat of solid argon. J Low Temp Phys, 1970, 3: 23-28

24 Keeler G J, Batchelder D N. Measurement of the elastic constants of argon from 3 to 77 K. J Phys C Solid State, 1970, 3: 510-522

Open Access This article is distributed under the terms of the Creative Commons Attribution License which permits any use, distribution, and reproduction in any medium, provided the original author(s) and source are credited. 